\title{
A prospective trial of CONfocal endomicroscopy in CYSTic lesions of the pancreas: CONCYST-01
}

\section{(ㄷ)(요 $\odot$}

\author{
Authors \\ Margaret G. Keane ${ }^{1}$, Natascha Wehnert ${ }^{1}$, Miguel Perez-Machado ${ }^{1}$, Giuseppe K. Fusai ${ }^{1}$, Douglas Thorburn ${ }^{1}$, \\ Kofi W. Oppong ${ }^{2}$, Nicholas Carroll ${ }^{3}$, Andrew J. Metz ${ }^{4}$, Stephen P. Pereira ${ }^{1}$
}

\section{Institutions}

1 Institute for Liver and Digestive Health, University College London, Royal Free Campus, Pond St, London, UK

2 Department of Gastroenterology and HPB Unit, Freeman Hospital, Newcastle upon Tyne, UK \& Institute of Cellular Medicine Newcastle University, Newcastle upon Tyne, UK

3 Cambridge University Hospital NHS Foundation Trust, Cambridge, UK

4 Department of Gastroenterology, Royal Melbourne Hospital, Parkville VIC, Australia

submitted 4.2.2019

accepted after revision 2.5.2019

\section{Bibliography}

DOI https://doi.org/10.1055/a-0957-2976 |

Endoscopy International Open 2019; 07: E1117-E1122

(c) Georg Thieme Verlag KG Stuttgart · New York

eISSN 2196-9736

Corresponding author

Professor Stephen Pereira, UCL Institute for Liver and Digestive Health, Royal Free Campus, Pond St, London, NW3 2PF, UK

Fax: +442079356826

stephen.pereira@ucl.ac.uk

\section{ABSTRACT}

Background and study aims Pancreatic cystic lesions $(\mathrm{PCL})$ are common. While some harbor malignant potential, accurate preoperative diagnosis remains challenging. Needle-based confocal laser endomicroscopy (nCLE) via a 19G FNA needle enables real-time imaging of the cyst wall. This study evaluated the safety and utility of nCLE in patients with an indeterminate $\mathrm{PCL}$ undergoing EUS-FNA.

Patients and methods The CONCYST study prospectivelyrecruited patients with indeterminate $\mathrm{PCL}$ attending three hepatopancreaticobiliary (HPB) referral centers in the UK, with indeterminate PCL, who required EUS-FNA between July 2014 and October 2016. Following the procedure, all patients were followed up in telephone clinic for at least 12 months. Ethical approval for the study was granted by the National Research Ethics Service (14/LO/0040).

Results Sixty-seven patient were recruited, 11 excluded and 56 included in the final analysis: 35 male, 21 female; median age 68 (range 28-80). Recognizable confocal images were obtained in 48 of 56 cases. Median nCLE scanning time was 5 minutes and did not exceed 10 minutes in any case. EUS-nCLE findings correlated with final diagnosis (based on imaging, cytology and multidisciplinary team review) in 43/56 (77\%) of cases, compared with 37/56 (66\%) for cytology alone $(P=0.12)$. One patient experienced mild pruritus following the procedure and another developed an infected pseudocyst, which resolved with antibiotics.

Conclusions EUS-nCLE under conscious sedation in the day case setting is safe and provides additional information to standard EUS-FNA for diagnosing indeterminate PCL.

\section{Introduction}

Pancreatic cystic lesions (PCL) have become an increasingly common finding, present in $1.2 \%$ to $2.6 \%$ of patients $[1,2]$ undergoing abdominal computed tomography (CT) and in up to $13.5 \%$ of patients undergoing magnetic resonance imaging (MRI) for non-pancreatic indications [3]. A PCL has a broad differential diagnosis [4]. In accordance with international and European guidance, patients with $\mathrm{PCL}$ that are thought to be malignant or that are at high risk of malignant transformation are referred for immediate surgical resection while other patients undergo regular surveillance with interval imaging [5]. However, every year, a proportion of patients with completely benign disease undergo unnecessary pancreatic resection as accurate differentiation of high-risk mucinous lesions preoperatively is a recognized clinical challenge. Not being able to differentiate benign lesions confidently also means growing numbers of patients are entering long-term surveillance [6]. 
The revised 2012 International Consensus guidelines recommend that endoscopic ultrasound (EUS) be performed in all suspected intraductal papillary mucinous neoplasm (IPMN) with worrisome features or when $>2 \mathrm{~cm}$ and surveillance is advocated [7]. The 2018 European consensus guidelines recommended performing EUS as an adjunct to other imaging modalities when results of EUS fine-needle aspiration (EUS-FNA) are expected to change clinical management [8].

Confocal laser endomicroscopy ( $\mathrm{nCLE}$ ) can provide real-time optical histology of the cyst wall, during EUS-FNA. A laser transmits a low-power laser beam via a probe within the FNA needle, to be focused onto cyst wall tissue and subsequently allows detection of fluorescent light, which is returned to the operating system to form the image. The probe used to image $P C L$ is the AQ-Flex miniprobe (Cellvizio; Mauna Kea Technologies, Paris, France), which can be passed down a 19G FNA needle during EUS (EUS-nCLE).

Initial studies to date of EUS-nCLE have found it to be a safe adjunct to routine EUS-FNA and have established diagnostic criteria for many of the common PCL. Although specificity was shown to be consistently high (>80\%) reported sensitivity has varied by cyst subtype and is generally lower for mucinous cydts (59\% to $95 \%$ for IPMN and $67 \%$ to $95 \%$ for mucinous cystic neoplasm) compared to serous cysts (69\% to $95 \%$ for serous cystic neoplasms [SCN]) [9-13]. Subsequent studies have also shown that there is good inter- and intra observer agreement [14]. To date the technique has not been evaluated in a UK population with indeterminate cystic lesions a; Phase II study was conducted to assess the safety and utility of this technology in this population (CONCYST-01).

\section{Patients and methods}

\section{Study aim}

The study aim was to define the safety and efficacy of nCLE in diagnosis of indeterminate PCL.

\section{Study design and inclusion criteria}

This phase II prospective study was conducted in three large regional HPB centers in the UK: Royal Free NHS Foundation Trust, London, Cambridge University NHS Trust, Cambridge, and Freeman Hospital, Newcastle.

Patients were included if they had a PCL for which EUS-FNA was indicated, based on multidisciplinary (MDT) review of cross-sectional imaging. The $\mathrm{PCL}$ had to be $>1 \mathrm{~cm}$. Patients had to be $>18$ years, with an Eastern Cooperative Oncology Group performance status 0,1 or 2 , an estimated life expectancy of at least 12 weeks and capable of giving written informed consent. They should not have had pancreatitis within the previous 3 months and women of child-bearing potential should have had a negative pregnancy test in the week before nCLE.

\section{Data recorded}

The electronic medical records of the included patients were reviewed and information was recorded in an electronic spreadsheet. Data collected included demographic information (age, sex, hospital number), initial symptoms, and history of acute or chronic pancreatitis or malignancy, family history of pancreatic cancer or relevant clinical syndrome. Cross-sectional imaging (computed tomography (CT) and/or magnetic resonance cholangiopancreatography [MRCP]) was obtained from PACS (picture archiving and communication system, GE Healthcare, United States) and relevant features recorded. Details of the endoscopic procedure were obtained from the gastrointestinal reporting tool. Pathology reports including cytology were obtained from the electronic histology database, in each center. For patients ultimately referred for surgery, date of the operation, type of resection and final histology were recorded. Length of follow-up was calculated from first procedure to last clinic appointment attended, or date of clinic discharge, or death.

\section{Definitions of PCL subtype by EUS-nCLE}

Definitions used in this study were established from previous EUS-nCLE publications ( $>$ Fig. 1).

Intraductal papillary mucinous neoplasm (IPMN): papillary projections $[9,10,15]$.

Serous cystic neoplasm (SCN): superficial vascular network (SVN) $[9,10]$.

Mucinous cystic neoplasm (MCN): the epithelial cyst border appears as a gray band delineated by a thin dark line $[9,10]$.

Pseudocyst: a pseudocyst was identified by bright, gray and black particles $[9,10]$.

Pancreatic cancer (pc): pc was identified by the presence of black clumps $[9,10]$.

Cystic pancreatic neuroendocrine tumor (PNET): Dark irregular clusters of cells, surrounded by various quantities of gray tissue $[9,10]$.

Indeterminate PCL: Lesions that after review of nCLE images did not display recognisable features of any of the PCL listed above.

\section{Final diagnosis}

Final diagnosis was based on pathology in those undergoing surgical resection. In all others, final diagnosis was based on MDT consensus with at least 12 months follow up.

\section{Procedures}

Endoscopic ultrasound-guided needle based confocal laser endomicroscopy (EUS-nCLE)

Informed written consent for the procedure and study participation was obtained. The procedures were performed under conscious sedation or general anaesthesia using a linear array echoendoscope (Olympus, UK or Hitachi Pentax). Once the cyst had been visualized from the stomach or duodenum, patients received $2.5 \mathrm{~mL}$ of $10 \%$ fluorescein. The cyst was then punctured with a 19G FN) needle (Cook Medical or Boston Scientific) which had been preloaded with the AQ-flex 19 miniprobe (Mauna Kea Technologies, Paris, France). Once in the cyst, the probe was gently advanced past the bevel of the needle and onto the cyst wall to begin nCLE imaging. Once the nCLE imaging had been completed, the probe was removed from the FNA needle and the cyst aspirated to dryness. Cyst 


\section{PCL}

IPMN:
papillary projections

\section{Pathology}

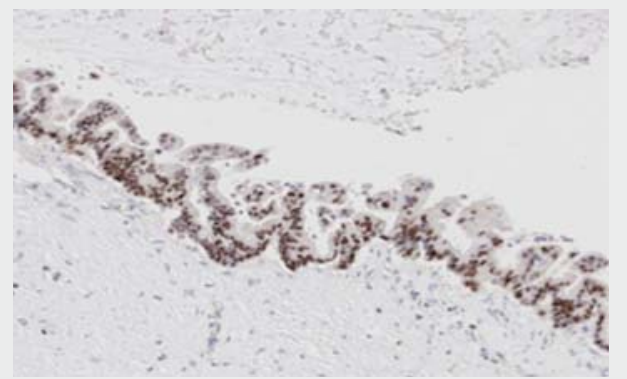

\section{SCN:}

superficial vascular network disorganised appearance with black clumps

Pseudocyst:

fibrous strands and debris
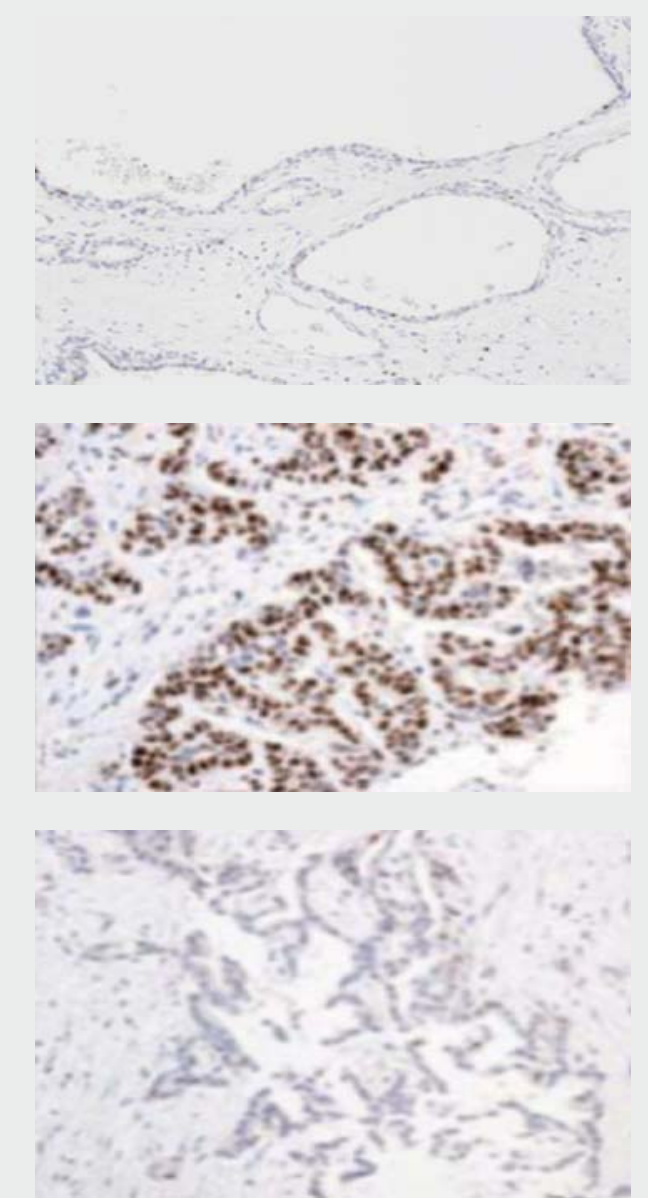

nCLE image
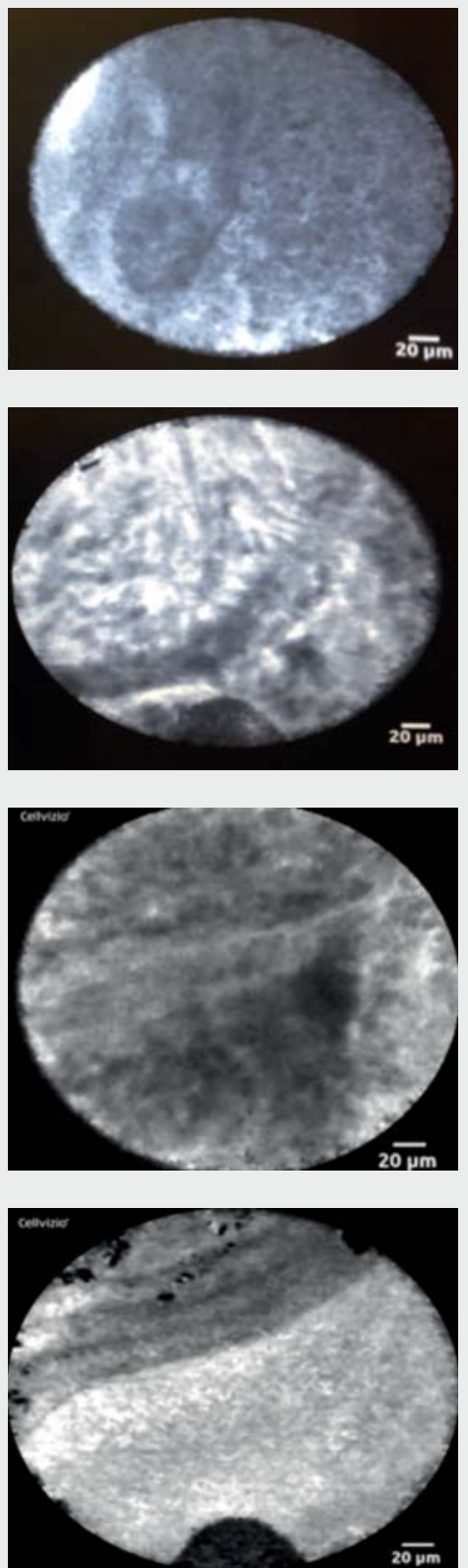

- Fig.1 nCLE findings for common PCL, compared to histopathology.

fluid was sent for cytology, fluid CEA, fluid amylase levels or gram stain and culture as clinically indicated. Patients were discharged within 4 hours from the recovery unit as long as they were clinically stable. A single dose of antibiotics was given to each patient during the procedure. Patients were then followed up by telephone clinic at 1 month and then seen as per routine in outpatients. Clinical records were reviewed at 12 months to confirm clinical outcome and all patient were discussed at the HPB multidisciplinary team (MDT) meeting following EUS to determine cyst subtype and the subsequent management plan.

\section{Ethical approval and consent to participate}

The CONCYST-01 study protocol was approved by the UK National Health Research Authority (14/LO/0040) and all patients gave written informed consent. The protocol was registered on ClinicalTrials.gov (13/0572).

\section{Data analysis}

Statistical Package for Social Sciences for Windows, version 22.0 (SPSS Inc., Chicago, Illinois, United States) was used to perform all statistical analyses. Associations between various 
clinical and radiographic characteristics were evaluated using a two-sample $t$ test for continuous variables, and a Chi-squared test for categorical variables.

The study sample size was based on cytology being diagnostic in $31 \%$ of cases of PCL [16] and nCLE in between $59 \%$ and $91 \%$ of cases, based on previous studies [9-13]. Assuming, a $5 \%$ significance level and $85 \%$ power, it was calculated that 61 patients were required for the study.

\section{Results}

Sixty-two patient were recruited during the study period; six were excluded, one because the cyst could not be visualized at EUS, one because of a gastric residue despite fasting making it unsafe to proceed with EUS under conscious sedation, two cases because the cyst could not be punctured with a 19G FNA needle, one because the lesion was determined to be solid at EUS, one case because of extravasation of the fluroscein.

Of the 56 patients included in the final analysis, 30 were male and 26 female; median age 68 (range 28-80). Twenty four of 42 patients ( $43 \%$ ) were symptomatic and in the remaining cases, the lesions were found incidentally. Eleven of 42 patients $(26 \%)$ had a history of pancreatitis. One patient had known von Hippel Lindau syndrome but no others had a family history of pancreatic cancer or associated syndromes. Seven patients had a history of a non-pancreatic solid organ malignancy. One patient had previously undergone a Whipple procedure for a 3-cm SCN. During assessment all patients had had a CT scan, 20 of 56 had an MRI and five of 56 patients had had an EUS (with indeterminate findings) prior to entering the CONCYST-01 study. Thirty-six percent of cysts were in the head or uncinate of the pancreas with the remaining lesions in the body or tail ( $\triangleright$ Table 1 ).

Final diagnosis was determined by post-surgical pathology in three cases and MDT consensus and follow up in all other cases ( $\triangleright$ Table 2 ). Four of 56 cases were lost to follow up. The remaining cases were followed up for $>12$ months with a review of the patient record at 12 months.

Determining diagnosis by clinical history and radiology alone performed poorly in comparison to EUS with cytology or EUS with nCLE (5.36\% vs. $66 \%$ vs. $77 \% P=0.001)$. Most cases, unless they had undergone malignant transformation, remained indeterminate after cross-sectional imaging, warranting further investigations.

Recognizable confocal images were obtained in 48 of 56 cases. Median nCLE scanning time was 5 minutes and did not exceed 10 minutes in any case. EUS-nCLE findings correlated with final diagnosis (based on imaging, cytology and multidisciplinary team review) in 43 of $56(77 \%)$ of cases, compared with 37 of 56 (66\%) for cytology alone ( $P=0.12)$. In IPMN cases, nCLE performed significantly better than routine cytology ( $90 \%$ vs. $69 \%, P=0.049$ ) ( $>$ Table 3 ). EUS-nCLE had an overall sensitivity of $79.6 \%$, which improved to $90 \%$ for IPMN, and $100 \%$ for pancreatic ductal adenocarcinoma; PDAC ( $\triangleright$ Table 4). When enough cyst fluid was obtained to measure fluid CEA, it was only diagnostic in cases with positive cytology so was not compared separately.
- Table 1 Patient demographics

\begin{tabular}{|c|c|c|}
\hline Patients & $\%$ & $\mathbf{n}$ \\
\hline Median age (range), years & $68(28-80)$ & \\
\hline \multicolumn{3}{|l|}{ Sex } \\
\hline - Male & $54 \%$ & $30 / 56$ \\
\hline - Female & $46 \%$ & $26 / 56$ \\
\hline \multicolumn{3}{|l|}{ Cyst morphology } \\
\hline \multicolumn{3}{|l|}{ Location } \\
\hline - Head & $32 \%$ & $18 / 56$ \\
\hline - Uncinate & $4 \%$ & $2 / 56$ \\
\hline - Body & $25 \%$ & $14 / 56$ \\
\hline - Tail & $39 \%$ & $22 / 56$ \\
\hline - Median lesion size (range), mm & $25(10-70)$ & \\
\hline - Associated symptoms & $43 \%$ & $18 / 42$ \\
\hline - Solid component/mural nodule & $16 \%$ & $9 / 56$ \\
\hline - Septations & $34 \%$ & $19 / 56$ \\
\hline - Dilated MPD (>5 mm) & $29 \%$ & $16 / 56$ \\
\hline - Median serum CA199 & 11.5 & \\
\hline \multicolumn{3}{|l|}{ Final diagnosis definition } \\
\hline - Surgery & $5 \%$ & $3 / 56$ \\
\hline - MDT consensus/cytology & $95 \%$ & $53 / 56$ \\
\hline
\end{tabular}

- Table 2 Final diagnosis (based on surgical resection or MDT consensus).

\begin{tabular}{|l|c|}
\hline Final diagnosis & $\mathbf{n}$ \\
\hline PDAC & 3 \\
\hline SB IPMN & 26 \\
\hline MD IPMN & 2 \\
\hline Multifocal IPMN + LGD & 1 \\
\hline PNET & 1 \\
\hline GIST & 1 \\
\hline Pseudocyst & 12 \\
\hline SCN & 9 \\
\hline Indeterminate cystic lesion & 1 \\
\hline $\begin{array}{l}\text { PDAC, pancreatic ductal adenocarcinoma; SB, side branch; IPMN, intraductal } \\
\text { papillary mucinous neoplasm; LGD, low-grade dysplasia; PNET, pancreatic } \\
\text { neuroendocrine tumor; GIST, gastrointestinal stromal tumor; SCN, serous } \\
\text { cystic neoplasm }\end{array}$ \\
\hline
\end{tabular}

The rate of associated AEs was 3.5\%. One patient experienced mild pruritus immediately after the procedure (probable allergy to fluorescein) and another developed an infected pseu- 
- Table 3 Comparing diagnostic accuracy of EUS nCLE to clinical history, radiology and EUS + cytology.

\begin{tabular}{|c|c|c|c|c|c|}
\hline Cyst subtype & $\begin{array}{l}\text { EUS nCLE vs. } \\
\text { final diagnosis }\end{array}$ & $\begin{array}{l}\text { History + radiology } \\
\text { vs. final diagnosis }\end{array}$ & $P$ value & $\begin{array}{l}\text { EUS + cytology } \\
\text { vs. final diagnosis }\end{array}$ & $P$ value \\
\hline All & $77 \%(43 / 56)$ & $5.36 \%(3 / 56)$ & $P<0.001$ & $66 \%(37 / 56)$ & 0.199 \\
\hline IPMN & $90 \%(26 / 29)$ & $0 \%(0 / 29)$ & $P<0.001$ & $69 \%(20 / 29)$ & 0.049 \\
\hline SCN & $56 \%(5 / 9)$ & $0 \%(0 / 9)$ & $P<0.001$ & $44 \%(4 / 9)$ & 0.621 \\
\hline Pseudocyst & $67 \%(8 / 12)$ & $0 \%(0 / 12)$ & $P<0.001$ & $92 \%(11 / 12)$ & 0.138 \\
\hline PDAC & $100 \%(3 / 3)$ & $100 \%(3 / 3)$ & - & $67 \%(2 / 3)$ & 0.322 \\
\hline
\end{tabular}

- Table4 Sensitivity, PPV and NPV for EUS nCLE by cyst subtype.

\begin{tabular}{|l|l|l|l|}
\hline $\begin{array}{l}\text { Cyst } \\
\text { subtype }\end{array}$ & $\begin{array}{l}\text { Sensitivity } \\
(\%)\end{array}$ & $\begin{array}{l}\text { Accuracy } \\
(\%)\end{array}$ & $\begin{array}{l}\text { PPV } \\
\text { (\%) }\end{array}$ \\
\hline $\begin{array}{l}\text { All PCL } \\
\text { (subtype) }\end{array}$ & $\begin{array}{l}79.63 \\
(66.47-89.37)\end{array}$ & $\begin{array}{l}76.79 \\
(63.58-87.02)\end{array}$ & $\begin{array}{l}95.56 \\
(94.95-96.09)\end{array}$ \\
\hline IPMN & 89.66 & 86.67 & 96.3 \\
& $(72.65-97.81)$ & $(69.28-96.24)$ & $(05.83-96.71)$ \\
\hline PDAC & 100 & 100 & 100 \\
\hline SCN & $(29.24-100)$ & $(29.24-100)$ & \\
\hline Pseudocyst & 55.56 & 38.46 & 55.56 \\
\hline & $(21.20-86.30)$ & $(13.86-68.42)$ & $(41.07-69.16)$ \\
\hline & $(34.89-90.08)$ & $(34.89-90.08)$ & 100 \\
\hline
\end{tabular}

PPV, positive predictive value; NPV, negative predictive value; EUS, endoscopic ultrasound; $\mathrm{nCLE}$, needle-based confocal laser endomicroscopy; PCL, pancreatic cystic lesion; IPMN, intraductal papillary mucinous neoplasm; PDAC, pancreatic ductal adenocarcinoma; SCN, serous cystic neoplasm

docyst, which resolved with intravenous antibiotics and a short hospital admission.

No significant differences were seen in nCLE performance or AEs between the individual three centers in the study.

\section{Discussion}

Early experience of EUS-nCLE using the AQ-Flex probe has shown it to be a safe technique and a useful adjunct to EUSFNA $[9-11,17]$. In this study, nCLE was found to have diagnostic accuracy similar to routine cytology ( $77 \%$ vs. $66 \%$; $P=0.12$ ). Although there was a trend towards statistical significance, our final patient number $(n=56)$ was smaller than the planned 61 patients (six cases were excluded for clinical or technical reasons), and diagnostic accuracy of cytology in this study was substantially higher than that reported in previous retrospective studies from our center or other published series (31\% diagnostic). Improvement in the accuracy of cytology in this study may be attributable to the cytopathologist being present in the endoscopy room for a proportion of the cases, ensuring the slides were prepared correctly and assessed immediately.
The study used the criteria defined by the international INSPECT, CONTACT and DETECT studies to identify cyst subtype [9-13]. These studies showed these criteria to have a high specificity (> $80 \%$ ) but a lower and somewhat variable sensitivity [9-13]. In this study we had similar findings with sensitivities ranging between $55 \%$ and $100 \%$. Somewhat unexpectedly, sensitivity of SCN in this study was only $55 \%$ which is lower than that reported in the CONTACT 1 study (69\% sensitivity and $100 \%$ specificity) [9]. This may reflect the operator's learning curve or alternatively the technique used for performing nCLE. In the French CONTACT 1 study, a lower sensitivity for nCLE in SCN (69\%) was also observed [9]. In these cases, the probe was "brushed or walked" along the wall during imaging, possibly resulting in epithelium being dislodged. In the subsequent CONTACT 2 study, a different technique was used to obtain nCLE images, with operators placing the probe on two points on the cyst wall only. In this subsequent study, there was improved sensitivity for nCLE in SCN group (>95\%). A rise in cytology yield was also seen in the CONTACT 1 study, which may be due to the epithelium being dislodged, therefore improving yields [13]. In this study, the probe was used in a similar way to the CONTACT 1 study, so imaging technique may also explain our lower sensitivity in SCN and improved cytology findings. Importantly in this study in IPMNs, nCLE was significantly more accurate at detecting IPMNs than routine cytology. Arguably this is the most important group to detect because of their premalignant potential.

AEs in this study were low at $3.5 \%$, with one transient pruritis and one infected pseudocyst but no cases of acute pancreatitis. From the trials to date, the average rate of post-procedural acute pancreatitis in PCLs was 4.3\% [9-12]. The highest rate was seen in the DETECT study (6.6\%), which required longer needle access time as the technique was combined with Spyglass cystoscopy as well as nCLE imaging [11]. Increased AEs are potentially attributable to prolonged procedure time and manipulation within the cyst $[11,12]$.

Although this study further confirmed the safety profile and utility of $\mathrm{nCLE}$, the technology is expensive and its place in routine diagnostic algorithms needs to be further defined. A recent study by the CONTACT authors looked at the cost-effectiveness of this technology in a French population and the potential for EUS-nCLE to prevent unnecessary over-treatment or surveil- 
lance, especially in patients with an SCN. They found that EUSnCLE would reduce the rate of surgical intervention by $23 \%$, with four in 1000 patient deaths prevented due to unnecessary surgery. This study has not been done in a UK population, but even allowing for a lower diagnostic accuracy seen in SCN in this study, similar benefits and cost savings are likely.

In this study, substantial differences were not observed between different IPMN subtypes or levels of dysplasia. This may be due to the relatively small sample size of patients with primarily small lesions under surveillance, which would be expected to have low levels of dysplasia. Emerging reports suggest that different subtypes of IPMN may have different criteria when imaged by EUS-nCLE [12], which could have prognostic significance when assessing the cyst preoperatively. Further studies of nCLE in patients who ultimately undergo surgical resection therefore are needed.

\section{Conclusions}

These initial results are encouraging and suggest that EUS-nCLE under conscious sedation in the day case setting is safe and provides additional information beyond standard EUS-FNA for diagnosing indeterminate cystic lesions of the pancreas.

\section{Acknowledgements}

The study was supported by grants from the Fiorina and Royal Free Hospital charities (https://www.royalfreecharity.org/charities/pancreatic-cancer-projects). Part of the work was undertaken at $\mathrm{UCLH} / \mathrm{UCL}$, which receives a proportion of funding from the Department of Health's National Institute for Health Research (NIHR) Biomedical Research Centres funding scheme.

\section{Competing interests}

None

\section{References}

[1] Laffan TA, Horton KM, Klein AP et al. Prevalence of unsuspected pancreatic cysts on MDCT. Am J Roentgenol 2008; 191: 802 - 807

[2] Spinelli KS, Fromwiller TE, Daniel RA et al. Cystic pancreatic neoplasms: observe or operate. Annals Surgery 2004; 239: 651 -657; discussion 657-659
[3] Ahn JY, Seo DW, Eum J et al. Single-Step EUS-guided transmural drainage of pancreatic pseudocysts: analysis of technical feasibility, efficacy, and safety. Gut Liver 2010; 4: 524 - 529

[4] Adsay NV, Fukushima N, Furukawa T et al. Intraductal neoplasm of the pancreas. In: Bosman FT, Carneiro F, Hruban RH, Theise ND. WHO classification of tumors of digestive system. Lyon: WHO Press; 2010

[5] Tanaka M, Fernandez-del Castillo C, Adsay V et al. International consensus guidelines 2012 for the management of IPMN and MCN of the pancreas. Pancreatology 2012; 12: 183-197

[6] Khashab MA, Shin EJ, Amateau S et al. Tumor size and location correlate with behavior of pancreatic serous cystic neoplasms. Am J Gastroenterol 2011; 106: 1521 - 1526

[7] Aad G, Abajyan T, Abbott B et al. Search for magnetic monopoles in sqrt $[\mathrm{s}]=7 \mathrm{TeV}$ pp collisions with the ATLAS detector. Phy Review Letters 2012; 109: 261803

[8] European Study Group on Cystic Tumours of the Pancreas. European evidence-based guidelines on pancreatic cystic neoplasms. Gut 2018; 67: $789-804$

[9] Napoleon B, Lemaistre Al, Pujol B et al. A novel approach to the diagnosis of pancreatic serous cystadenoma: needle-based confocal laser endomicroscopy. Endoscopy 2015; 47: 26-32

[10] Jais B, Rebours V, Malleo G et al. Serous cystic neoplasm of the pancreas: a multinational study of 2622 patients under the auspices of the International Association of Pancreatology and European Pancreatic Club (European Study Group on Cystic Tumors of the Pancreas). Gut 2016; 65: 305-312

[11] Nakai Y, Iwashita T, Park do H et al. Diagnosis of pancreatic cysts: EUSguided, through-the-needle confocal laser-induced endomicroscopy and cystoscopy trial: DETECT study. Gastrointestinal endoscopy 2015; 81: $1204-1214$

[12] Kamboj AK, Dewitt JM, Modi RM et al. Confocal Endomicroscopy Characteristics of Different Intraductal Papillary Mucinous Neoplasm Subtypes. J Pancreas 2017; 18: $198-202$

[13] Napoleon B, Palazzo M, Lemaistre Al et al. Needle-based confocal laser endomicroscopy of pancreatic cystic lesions: a prospective multicenter validation study in patients with definite diagnosis. Endoscopy 2018. doi:10.1055/a-0732-5356

[14] Krishna SG, Brugge WR, Dewitt JM et al. Needle-based confocal laser endomicroscopy for the diagnosis of pancreatic cystic lesions: an international external interobserver and intraobserver study (with videos). Gastrointestinal endoscopy 2017; 86: 644-654.e642

[15] Khan I, Kumar N, Pant I et al. Activation of TGF-beta pathway by areca nut constituents: a possible cause of oral submucous fibrosis. PloS one 2012; 7: e51806

[16] de Jong K, Poley JW, van Hooft JE et al. Endoscopic ultrasound-guided fine-needle aspiration of pancreatic cystic lesions provides inadequate material for cytology and laboratory analysis: initial results from a prospective study. Endoscopy 2011; 43: 585-590

[17] Konda V], Aslanian HR, Wallace MB et al. First assessment of needlebased confocal laser endomicroscopy during EUS-FNA procedures of the pancreas (with videos). Gastrointestinal endoscopy 2011; 74: $1049-1060$ 